\title{
Symmetry and similarity effects in the comparison of visual patterns
}

\author{
SEBASTIANO BAGNARA \\ Institute of Psychology, CNR, Rome, Italy \\ DAVID B. BOLES \\ University of Oregon, Eugene, Oregon \\ FRANCESCA SIMION \\ University of Padua, Padua, Italy \\ and \\ CARLO UMILTA \\ University of Parma, Parma, Italy
}

\begin{abstract}
Models to explain same-different RT disparity have variously emphasized encoding or comparison processes. Of the latter, a dual-process holistic-analytic model that bears a similarity to a putative distinction between hemispheric processes has been proposed. Here two experiments test the models by employing simultaneous and successive matching while varying letter similarity, symmetry, and visual field of presentation. Same-different disparity is found regardless of delay, although it can be eliminated with appropriate similarity and symmetry manipulations. While visual field interacts with the same-different factor, it fails to do so in a manner consistent with the proposed hemispheric dichotomy. These and other results argue against encoding models of letter matching, and support a revised visual comparison model that incorporates dual processing and response criterion elements. However, the role of lateralized processes remains unclear.
\end{abstract}

The act of comparing two things and determining whether they are the same or different is basic in mental activity. Empirically, a major observation has been that the time taken to judge letters as physically the same is often less than the time taken to establish that they are different (Nickerson, 1972, 1978). This finding has led to two classes of models attributing the same-different disparity either to the comparison or to the encoding processes.

The model put forward by Proctor (1981) is representative of those stressing the importance of encoding processes. According to Proctor, letter comparison regardless of case (e.g., AA or Aa) always involves the name code (Posner, 1978), at least when successive matches are considered, and it is assumed that the first item can act as a "prime" for the second. When the two items are the same, the prime is valid, and then the process of encoding the second item is facilitated by the already activated pattern. When the two items are different, the prime is invalid and the second letter's encoding gains no beneficial effect. On the contrary, a conflict between activated

This research was partially supported by National Institute of General Medical Science Grant 5T01 GM02165. Reprint requests should be addressed to David B. Boles, Department of Psychology, University of Illinois, Champaign, Illinois 61820 . patterns ensues, bringing about inhibition and, thus, slow reaction times.

In contrast, models stressing the importance of comparison process in determining same-different disparity usually invoke a two-process method of comparison with separable processes underlying the possible responses. Typically, a fast, holistic process (identity reporter) is assumed to underlie "same" responses, while a slow analytic self-terminating process is assumed to underlie "different" responses (Bamber, 1969; Hock, 1973; Taylor, 1976).

Both of these models are subject to criticism. The encoding account, for example, strictly speaking applies only to successive matches (when priming can operate). Nevertheless, although Proctor (1981) argues that the same-different disparity occurs only for successive matches, it is clear that in some instances the disparity appears in simultaneous matches and so must be accounted for (Bagnara, Boles, Simion, \& Umilta, 1982; Bindra, Donderi \& Nishisato, 1968; Royer, 1981). In addition, Bagnara et al. reported evidence that a visual code can be used in successive matching, constituting an additional problem for Proctor's account. On the other hand, the two-process account has been criticized for lack of parsimony (Knoll \& Hersherson, 1980; Proctor, 1981) and inadequate consideration of errors (Krueger, 1978). 
Because of the latter criticisms, Krueger (1978) proposed a single-process comparison model to explain the same-different disparity. This model postulates that the perceiver bases both positive and negative judgments on how many mismatching features a "difference counter" records. However, since internal noise may produce a positive difference count even for same pairs, all positive counts must be rechecked to assure that the counted mismatches are not due to noise. As a result, all "different" responses are delayed by rechecking, while some "same" responses (those resulting from a zero count) are not. Rechecking thus accounts for the same-different disparity.

One difficulty for Krueger's account arises from the converse observation to the one problematic for Proctor: in some instances, "same" responses are not reliably shorter than "different" responses (Bindra et al., 1968; Proctor, 1981). This poses a problem for a model in which all positive "difference counts" are rechecked, although a revised model could include criterial shifts to account for the observation (cf. Nickerson, 1981). Such a revision might state that rechecking is not performed when different letter pairs are so dissimilar that internal noise is insufficient to make same pairs mimic them in difference counts.

All things considered, it seems apparent that additional evidence is needed before a conclusion can be reached on the mechanism responsible for samedifferent disparity. In this paper, the results of a number of potentially informative manipulations are reported. First, on the most basic level, simultaneous and successive presentations are both employed to determine the conditions under which a same-different disparity can reliably be found. If the disparity is found even when presentations are simultaneous, as in fact has been reported previously (Bindra et al., 1968; Krueger, 1983), this would constitute evidence against Proctor's priming model. Second, visual similarity is varied to assess the effects of this variable on the disparity. If the disparity varies with visual similarity (Bindra et al., 1968), this would suggest either a two-process letter-comparison model or a single-process model combined with a criterion shift. Third, the use of symmetry as a "structural diagnostic" is investigated, since it has been found that symmetry can speed "same" responses (Fox, 1975; Richards, 1978), an observation unaccounted for in any of the preceding models. If found, such an effect would implicate a two-process comparison model, since symmetry should not in itself affect a difference count. A fourth manipulation, that of the visual field of presentation, is considered below.

\section{The Hemispheric View}

In the last decade, a major goal of hemispheric research has been the description and experimental support of elementary principles of hemispheric organization (Cohen, 1973; Egeth \& Epstein, 1972; Klatzky \& Atkinson, 1971; Martin, 1979; Patterson \& Bradshaw, 1975; Umilta, Salmaso, Bagnara, \& Simion, 1979). Such principles typically are given a dichotomous form, the one central to this report being that the right hemisphere can be described as a "holistic" or "global" processor and the left hemisphere as an "analytic" processor (Levy-Agresti \& Sperry, 1968; Patterson \& Bradshaw, 1975). Applied to vision, the distinction is fundamentally similar to that described above by comparison process theorists in nonhemispheric contexts (Bamber, 1969; Hock, 1973; Taylor, 1976).

Recently, we reported experimental tests of the hemispheric dichotomy employing the letter-matching task of Taylor (Bagnara et al., 1982). We showed, as did Taylor, that $\mathrm{RT}$ for different responses depended on the quantified number of visual differences between letters, implying a serial analytic process. Furthermore, "same" responses were faster than "different" matches, a result inconsistent with the analytic process but suggesting a fast holistic match. Although these results appeared to operationalize the dichotomy, tests of visual-field (VF) asymmetry for "same" and "different" responses produced no evidence that the right and left hemispheres were holistic and analytic processors, respectively.

Patterson and Bradshaw (1975), however, have outlined a position contrasting with the notion that all different letter pairs are matched analytically. On the basis of face-matching experiments, they proposed that if discriminanda are made sufficiently different, a right-hemisphere holistic process is capable of detecting differences and thus of producing fast RTs. To further complicate matters, a third position appears in the hemispheric literature which sharply contrasts with both of these accounts. By this view, the right hemisphere is a generalized "visuospatial" processor, and its advantage in processing "visuospatial" stimuli enlarges with increasing similarity among discriminanda. The rationale given for such an effect varies, but two suggestions have been that increasing similarity (1) in effect emphasizes right-hemisphere visual processors (Hellige \& Webster, 1979), or (2) decreases the probability of verbal encoding of stimuli (Berlucchi, 1974).

In the present research, visual field is a fourth manipulated variable intended to address these issues by allowing inferences about hemispheric processes. According to the dual-process hypothesis advanced by Taylor and extended by Bagnara et al. (in conjunction with a holistic-analytic hemispheric dichotomy), visual field should interact with the samedifferent factor. In contrast, the other two viewpoints predict visual field by degree of difference interactions of opposing forms (neither of which, however, 
were found by Bagnara et al.). In addition, the investigation of potential field $\times$ symmetry interactions brings another approach to bear on the putative hemispheric dichotomy, since the extraction and use of symmetry to speed responses must represent a process that is neither strictly holistic (it involves comparing areas within letters) nor strictly analytic (it combines information across areas).

In Experiment 1, simultaneous letter matching was investigated.

\section{EXPERIMENT 1}

\section{Method}

Subjects. Sixteen subjects, eight of each sex, participated in the experiment. They were drawn from the University of Oregon Cognitive Laboratory subjeci pool, and were paid for the 1-h session. All were right-handed.

Apparatus. Stimuli and performance feedback were presented on a Hewlett-Packard 1300A X-Y display CRT controlled by a PDP-15 computer. Responses were made on a two-key board in front of the subject.

Stimuli. The "same"-pair stimuli were the capital letter pairs $\mathrm{HH}, \mathrm{MM}, O O$, and UU (all bilaterally symmetrical) and CC, GG, $\mathrm{NN}$, and QQ (all bilaterally asymmetrical). There were two sets of "different" pairs. Visually confused "different" pairs were CG, CQ, GO, HN, HU, MN, MU, and OQ, which have a mean confusion frequency of $17.3(s=19.8)$ in Hodge's $(1962)$ study. (Podgorny \& Garner, 1979, have shown confusability to be a reliable measure of similarity.) Nonconfused "different" pairs were $\mathrm{CH}, \mathrm{CU}, \mathrm{GM}, \mathrm{GN}, \mathrm{HO}, \mathrm{MQ}$, NO, and UQ, with a mean confusion frequency of $0.0(s=0.2)$. Pairs were presented equal numbers of times in both orders (e.g., UQ and QU), so both "different"pair sets used the same letters in the same positions, differing only in pairings. The letters were $10 \times 7 \mathrm{~mm}$ in height and width.

Procedure. A trial had the following order of events: (1) a pair of vertical fixation dots appeared centrally for $1,000 \mathrm{msec}$, together subtending $2 \mathrm{~mm}$ vertically $\times$ less than $1 \mathrm{~mm}$ horizontally; (2) a letter pair appeared for $140 \mathrm{msec}$ in one visual field or the other, in either a horizontal array $(10 \times 20 \mathrm{~mm}$, with near end $31 \mathrm{~mm}$ from fixation) or a vertical array $(26 \times 7 \mathrm{~mm}$, with near end $37 \mathrm{~mm}$ from fixation); (3) a blank screen remained until the response or 1,000 msec had passed; (4) RT and accuracy feedback was displayed for $200 \mathrm{msec}$; and (5) a blank screen again remained for $1,000 \mathrm{msec}$, the intertrial interval.

The subjects responded "same" with the index finger of one hand and "different" with the opposite index finger. Hand mapping was counterbalanced across subjects. RTs longer than an arbitrary limit of $1,140 \mathrm{msec}$ were considered errors.

The subjects first received a practice block of 64 trials: half were "same" and half were "different," half were horizontal arrays and half were vertical ones, half were LVF and half were RVF. This was followed by six experimental blocks of the same length and structure. For eight subjects, these seven blocks contained visually confused "different" pairs, while the other eight received nonconfused pairs. In either case, they were followed by seven more blocks (the first one practice) containing the other pairs.

Instructions stressed the importance of keeping the eyes fixed on the central fixation dots, and gave a goal of about $10 \%$ errors. The subjects were allowed to view the display at self-selected distances, so it is not possible to specify dimensions in visual angle with any precision. The rationale for this procedure is that visualfield asymmetry appears to be independent of eccentricity, as long as displays are symmetric around central fixation (Harvey, 1978; Haun, 1978).

\section{Results}

Because symmetry pertains only to "same" pairs, two analyses were required. In the first, RT and errors were entered into ANOVAs, excluding the symmetry factor; in the second, symmetry effects were assessed for same responses alone.

RT. The overall RT data were subjected to a match (same vs. different) $\times$ visual field (LVF vs. RVF) $X$ similarity of different pairs (similar vs. dissimilar) ANOVA. The three-way interaction is shown in Table 1.

There were four significant terms. Of the main effects, match and similarity were significant $[F(1,15)$ $=22.08, \mathrm{p}<.001$, and $F(1,15)=30.44, \mathrm{p}<.001]$. "Same" responses were faster than "different" responses (582 vs. $610 \mathrm{msec}$ ), and blocks in which "different" pairs were dissimilar produced faster RTs than blocks containing similar "different" pairs (555 vs. $637 \mathrm{msec}$ ).

The other significant terms were two-way interactions: match $\times$ visual field $[F(1,15)=5.21, p<.05]$ and match $\times$ similarity $[F(1,15)=15.14, p<.005]$. Both are apparent from Table 1. Although individually nonsignificant, there was a tendency for the RVF to be faster than the LVF for "same" matches (572 vs. $592 \mathrm{msec}$ ), and a reverse tendency for "different" matches (616 vs. $604 \mathrm{msec}$ ). Interestingly, although identical "same" pairs were used in both block types, RT to "same" responses was affected by the similarity factor ( 550 vs. $615 \mathrm{msec}$, in dissimilar vs. similar blocks), although to a lesser extent than were the different responses themselves (560 vs. $660 \mathrm{msec}$ ).

Finally, it should be noted that the three-way interaction was not significant $[F(1,15)=1.21]$.

Errors. Errors were subjected to a similar ANOVA. Three factors were significant: similarity $[F(1,15)=$ $68.45, \mathrm{p}<.001]$, match $\times$ visual field $[F(1,15)=$ $4.86, \mathrm{p}<.05]$, and match $\times$ similarity $[\mathrm{F}(1,15)=$ $11.83, \mathrm{p}<.005]$. All were congruent with the corresponding RT terms. Thus, there were fewer errors in dissimilar than in similar blocks ( $7 \%$ vs. $18 \%$ ); there were tendencies toward fewer RVF than LVF errors to "same" pairs $(12 \%$ vs. $16 \%)$ and fewer LVF errors to "different" pairs ( $11 \%$ vs. $14 \%)$; and "same" errors were influenced by "different"-pair similarity ( $9 \%$ vs. $16 \%$ in dissimilar vs. similar blocks),

Table 1

The Match $\times$ Visual Field (in Milliseconds) $\times$ Similarity Interaction in Experiment 1 RTs

\begin{tabular}{lccccc}
\hline & \multicolumn{2}{c}{ Dissimilar } & & \multicolumn{2}{c}{ Similar } \\
\cline { 5 - 6 } & LVF & RVF & & LVF & RVF \\
\hline Same & 560 & 540 & & 624 & 605 \\
Different & 557 & 562 & 651 & 669 \\
\hline
\end{tabular}


although to a lesser extent than the "different" errors $(6 \%$ vs. $20 \%)$. The three-way term was not significant $[F(1,15)=2.63]$.

Symmetry effects. To investigate the role of symmetry in letter matching, "same" RTs were further analyzed in a symmetry (symmetrical vs. asymmetrical) $x$ orientation of letter pairs (horizontal vs. vertical) $x$ visual field $\times$ similarity of "different" pairs ANOVA. The three-way interaction of symmetry $x$ visual field $x$ similarity is illustrated in Table 2 .

Symmetry entered into three significant terms, the main effect of symmetry $[F(1,15)=69.02, p<.001]$ and the interactions of symmetry $x$ orientation $[F(1,15)=7.26, p<.025]$ and symmetry $\times$ similarity $[F(1,15)=10.28, p<.01]$. Bilaterally symmetrical pairs produced shorter RTs than asymmetrical pairs (558 vs. $610 \mathrm{msec}$ ). Importantly, this effect was larger in horizontal arrays (557 vs. $617 \mathrm{msec}$ ) than in vertical arrays ( 560 vs. $603 \mathrm{msec}$ ) and in similar blocks ( 587 vs. $650 \mathrm{msec}$ ) than in dissimilar blocks (530 vs. $569 \mathrm{msec}$ ). As Table 2 suggests, the symmetry $\times$ visual field interaction was not significant $[F(1,15)$ $=2.25$. .

In the corresponding error analysis, symmetry entered into four terms. The main effect $[F(1,15)=$ $48.15, \mathrm{p}<.001]$ showed that symmetrical letters produced fewer errors than asymmetrical letters $(8 \%$ vs. $20 \%$ ). In addition, the symmetry $\times$ orientation interaction $[F(1,15)=6.73, p<.025]$ agrees with the $R T$ analysis: the symmetry effect was larger in the horizontal pairs (8\% vs. $23 \%$ ) than in vertical pairs $(8 \%$ vs. $17 \%)$. It was also larger in similar blocks $(10 \%$ vs. $26 \%$ ) than in dissimilar blocks $(7 \%$ vs. $14 \%)$, as shown by the symmetry $\times$ similarity interaction $[F(1,15)=11.34, p<.005]$. Finally, the four-way interaction was significant $[F(1,15)=7.57, p<.025]$, but no interpretation is apparent.

\section{Discussion}

The results of Experiment 1 bear directly on the general and hemispheric models of letter comparison described in the introduction. First, the same-different disparity was obtained despite the fact that both letters in a pair were simultaneously presented, in confirmation of Bindra et al. (1968) and Krueger (1983). The advantage for "same" responses, although perhaps smaller than that usually obtained when successive presentations are employed (Nickerson, 1978), was highly significant. This outcome is not easily ac-

Table 2

The Symmetry $\times$ Visual Field (in Milliseconds) $\times$ Similarity Interaction in Experiment 1 "Same" RTs

\begin{tabular}{lccccc}
\hline & \multicolumn{2}{c}{ Dissimilar } & & \multicolumn{2}{c}{ Similar } \\
\cline { 2 - 3 } \cline { 5 - 6 } & LVF & RVF & & LVF & RVF \\
\hline Symmetrical & 536 & 524 & & 595 & 578 \\
Asymmetrical & 584 & 554 & 661 & 640 \\
\hline
\end{tabular}

commodated by Proctor's (1981) model, since the model posited that successive priming was responsible for the same-different disparity.

Second, it was found that the disparity was larger when "different" pairs were visually similar than when they were dissimilar (45 vs. $10 \mathrm{msec}$ ). This result supports the suggested modification of Krueger's (1978) model, in that it seems that the same-different disparity can be virtually eliminated by making "same" and "different" pairs sufficiently discriminable. If Krueger's model is modified with a criterial assumption, this result can be accommodated as the product of internal noise's being insufficient to cause confusion in difference counts between "same" and dissimilar "different" pairs, thus averting the recounting process, which presumably accounts for same-different disparity. Additional evidence supporting this comes from the analysis of symmetry effects on "same" pairs, where it can be shown that the disparity also disappears when asymmetric sames are compared with differents (RTs to both were $610 \mathrm{msec})$.

Third, the obtained effects of symmetry introduce further constraints to be placed on general models of letter comparison. Experiment 1 clearly supports a role of symmetry in speeding responses, as did the results of Royer (1981) in a different context, and it demonstrates separable effects of letter and display symmetry. Bilaterally symmetrical letter pairs exerted a larger $(60 \mathrm{msec})$ effect when arranged horizontally than when arranged vertically $(43 \mathrm{msec})$. Since the letters used in both instances were identical, this difference cannot be attributed to letter symmetry, but rather must be accounted for by display symmetry. Yet, vertical arrangements did produce a nonzero advantage of symmetry, even when top-bottom symmetrical letters ( $\mathrm{H}$ and $\mathrm{O}$ ) are excluded in favor of purely bilaterally symmetrical letters $(M$ and $U ; t(15)$ $=5.31, \mathrm{p}<.001]$. This indicates that letter, as well as display, symmetry is effective in speeding "same" responses.

Finally, it is noteworthy that the effect of symmetry was larger when "different" pairs were similar than when they were dissimilar (63 vs. $39 \mathrm{msec}$ ). This is supportive of symmetry as a "diagnostic" allowing fast "same" responses (Fox, 1975), since a diagnostic would be expected to become more beneficial as similarity increased. In effect, the presence of similar "different" pairs should make subjects cautious and thus slow in making same responses; symmetry can then exert a larger diagnostic effect than otherwise. By contrast, the result is not very compatible with the view of Richards (1978), who suggested that symmetry plays its role in an initial field segmentation process. It would be difficult to explain an effect of "different" pair similarity with this mechanism. Also worthy of note in this respect is that field segmentation cannot account for the role of letter, as 
opposed to display, symmetry present in this experiment.

One significance of the symmetry effects is that they are incompatible with single-process models of generalized letter comparison (i.e., Krueger, 1978). Since symmetry is clearly functioning to produce very fast "same" responses, it cannot be that a difference counter is sufficient to produce same-different disparity in its entirety. Rather, there must be something special about "same" pairs that such diagnostics can speed responses to them, implicating a dual-process comparison model.

With regard to hemispheric theories of the matching task, the results of Experiment 1 give support to none of the three outlined in the introduction. Although visual field interacted with the same-different factor, it did so in a fashion contrary to the view that "same" matches are made holistically and "different" matches analytically, and by the right and left hemispheres, respectively. Nevertheless, the result is interesting, because it again argues against a singleprocess model of letter matching, demonstrating a dissociation of "same" and "different" matches by visual field.

As for the other two theories, Patterson and Bradshaw's (1975) account predicts that visually nonconfused "different" pairs should result in a LVF (right hemisphere) advantage, and confused pairs should result in a RVF (left hemisphere) advantage, yet Table 1 shows little evidence of such an effect, and no significant term in the analyses confirms it.

The "visuospatial" viewpoint predicts an increasing LVF advantage with increasing confusion, and, although the results were slightly in this direction (Table 1), again no significant term is in support.

Having examined the roles of similarity, symmetry, and visual field on simultaneous letter matches, we next considered successive matches. Experiment 2 was designed with this purpose in mind.

\section{EXPERIMENT 2}

\section{Method}

Subjects. A new sample of 16 subjects, with the same characteristics as in Experiment 1, participated.

Apparatus and Stimuli. The apparatus and stimuli employed in the previous experiment were used here.

Procedure. Each trial had the following order of events: (1) A single letter appeared at fixation for $500 \mathrm{msec}$, followed by (2) 50 msec of blank screen, (3) a pair of fixation dots for $1,000 \mathrm{msec}$, (4) the second member of the letter pair, in one visual field (with near edge $36 \mathrm{~mm}$ from fixation) for $140 \mathrm{msec}$, and (5) blank screen until the response was made or until 1,000 msec had passed, (6) 200 msec of feedback, and (7) blank screen for $1,000 \mathrm{msec}$, the intertrial interval.

In other respects, except for the absence of horizontal or vertical arrays as such, the procedure was identical to that of Experiment 1.

\section{Results}

RT and Errors. Mean RTs were entered into an ANOVA with the following factors, defined as in
Experiment 1: Match, visual field, and similarity. The three-way interaction is displayed in Table 3.

There were three significant terms. The main effect of match $[F(1,15)=80.77, p<.001]$ reflects faster responses for sames than for differents (486 vs. $525 \mathrm{msec}$ ). However, this factor interacted with both visual field $[\mathrm{F}(1,15)=6.42, \mathrm{p}<.025]$ and similarity $[F(1,15)=27.00, p<.001]$. Thus, there were tendencies for "same" responses to be faster to the RVF than to the LVF (480 vs. $493 \mathrm{msec}$ ) and vice versa for different responses ( 528 vs. $522 \mathrm{msec}$ ), although neither asymmetry was significant by itself. Furthermore, "same" matches were unaffected by dissimilarity versus similarity within different pairs (485 vs. $488 \mathrm{msec}$ ), although the latter were themselves affected (511 vs. $539 \mathrm{msec})$. There were no other significant effects, including the three-way interaction $(F<1)$. In a similar analysis of errors there were no significant terms.

Symmetry effects. As in Experiment 1, the same responses were further analyzed for the effect of bilateral symmetry. In RT, symmetry exerted a main effect $[F(1,15)=44.77, p<.001]$, with symmetrical letters producing faster responses than asymmetrical letters (472 vs. $502 \mathrm{msec}$ ). There was also a symmetry $x$ similarity interaction $[F(1,15)=35.81, p<.001]$, illustrated in Table 4 (in conjunction with the visual field factor), with symmetry showing a larger effect in similar blocks $(42 \mathrm{msec})$ than in dissimilar blocks $(18 \mathrm{msec})$. In errors, symmetry had no effect.

\section{Discussion}

The results of Experiment 2 are fundamentally similar to those of Experiment 1, extending many of the conclusions to the successive matching case. Again, same-different disparity was found, and the fact that it was of reasonably similar magnitude in the second experiment $(39 \mathrm{msec})$ and the first $(28 \mathrm{msec})$ implies that the effect of priming must be slight, a problem for Proctor's (1981) view. Although, taken alone, a comparison of disparity when "different" pairs were similar and dissimilar ( 51 vs. $26 \mathrm{msec}$ ) is not particularly suggestive that it could be eliminated if different pairs were sufficiently dissimilar, the disparity between asymmetric "same" and "different" pairs in dissimilar blocks is smaller yet $(17 \mathrm{msec})$ and does lend some support to the proposed modification of Krueger's model (cf. Bindra et al., 1968). And, again, visual factors were found to affect RT, with symmetry affecting "same" responses and similarity

Table 3

The Match $\times$ Visual Field (in Milliseconds) $\times$ Similarity Interaction in Experiment 2 RTs

\begin{tabular}{llllll}
\hline & \multicolumn{2}{c}{ Dissimilar } & & \multicolumn{2}{c}{ Similar } \\
\cline { 2 - 3 } \cline { 5 - 6 } & LVF & RVF & & LVF & RVF \\
\hline Same & 492 & 478 & & 494 & 482 \\
Different & 509 & 513 & & 536 & 542 \\
\hline
\end{tabular}


Table 4

The Symmetry $\times$ Visual Field (in Milliseconds) $\times$ Similarity Interaction in Experiment 2 "Same" RTs

\begin{tabular}{|c|c|c|c|c|}
\hline & \multicolumn{2}{|c|}{ Dissimilar } & \multicolumn{2}{|c|}{ Similar } \\
\hline & LVF & RVF & LVF & RVF \\
\hline Symmetrical & 482 & 471 & 475 & 459 \\
\hline Asymmetrical & 502 & 486 & 514 & 504 \\
\hline
\end{tabular}

affecting "different" responses. This finding is difficult to reconcile with Proctor's notion that all simultaneous letter matching is done according to a "name" code, as is the recently reported failure of phonetic confusion to affect both simultaneous and successive matches (Boles \& Eveland, 1983). Furthermore, the symmetry effects suggest, as they did in Experiment 1, that somewhat disparate processes underlie "same" and "different" matches.

Another argument for a dual-process method of comparison comes from the interaction of visual field with the same-different factor, suggesting a hemispheric dissociation of the two types of matches. However, the interaction was again incongruent with the putative holistic-analytic hemispheric dichotomy. Furthermore, visual field failed to interact with similarity or symmetry, arguing against the positions of Patterson and Bradshaw (1975) and of the "visuospatial" theorists (Berlucchi, 1974; Hellige \& Webster, 1979).

\section{GENERAL DISCUSSION}

The major findings of the two experiments are as follows. First, an overall same-different disparity can be found in both simultaneous and successive letter-matching tasks. Second, however, there are indications that the disparity can be eliminated if "same" pairs are asymmetric and "different" pairs are sufficiently dissimilar. Third, effects of symmetry and similarity show that visual representations are important, even when matching is successive. And fourth, visual field interacts with the same-different factor in that "same" and "different" responses tend to be faster in the right and left visual fields, respectively, although visual field does not interact with letter similarity or symmetry.

Together, these results argue against every one of the general and hemispheric models of letter comparison outlined in the introduction. Proctor (1981) argued that successive matching always involves the "name" code, yet the present clear support for visual factors makes such a position difficult. Similarly, the finding that same-different disparity can be found even for simultaneous matches questions Proctor's treatment of such matches as a special case, and challenges his encoding (priming) view of the effect.

On the other hand, problems are also posed for current comparison-process views. The idea that a fast holistic identity reporter underlies "same" matches, and a slow analytic process underlies "different" matches, suffers from the discovery that the same-different disparity can be eliminated by appropriate symmetry and similarity manipulations. In contrast to this dual-process view, Krueger's (1978) single-process model essentially claims that all matches are made by an analytic "difference counter," yet the observation that symmetry can dramatically speed "same" responses is problematic here. Since symmetry is an aspect of sameness, it is difficult to see how its presence could speed the matching of "same" pairs by a process analytically counting differences.

Nor do the hemispheric models fare better. The holistic-analytic view of hemispheric differences predicts a visual field by same-different interaction opposite to the one obtained, although it could be argued that the present paradigm failed to operationalize the dichotomy as implied above. Nevertheless, it is worth noting that the obtained interaction is similar to others reported elsewhere (Bagnara et al., 1982; Cohen, 1973; Egeth \& Epstein, 1972) and that alternative operationalizations of the putative hemispheric dichotomy have often failed to support it (Boles, Note 1; Fairweather, Brizzolara, Tabossi, \& Umilta, 1982). Finally, the views that decreasing (Patterson \& Bradshaw, 1975) or increasing the similarity of discriminanda (Berlucchi, 1974; Hellige \& Webster, 1979) shifts processing in favor of the right hemisphere were not supported by any interaction of visual field with similarity.

In spite of these negative implications, it is possible to propose a model of letter matching that can accommodate the results of Experiments 1 and 2. An initial consideration is that it must incorporate something like dual processing as a fundamental assumption, since otherwise it is difficult to simultaneously account for (1) the effect of symmetry in speeding "same" responses, (2) the demonstrated analytic nature of different responses (Bagnara et al., 1982; Bamber, 1969; Hock, 1973; Taylor, 1976), and (3) the observed visual field by response interaction (which implies a dissociation by hemisphere). Clearly, however, it would be more appropriate to label these processes "superfeatural" versus "featural," since, as argued above, a holistic-analytic distinction no longer appears tenable. "Featural" is taken as implying an analytic process operating at the feature level, while "superfeatural" is suggested as a description for the extraction of symmetry both within and between letters.

In addition, it seems desirable to include in the model a criterial assumption such as the modification proposed in the introduction for Krueger's theory. Perhaps, as Krueger proposed, the "analytic" (here "featural") process is indeed a difference counter, and same-different disparity can emerge in a rechecking of all nonzero difference counts. By making different pairs sufficiently dissimilar, however, the rechecking process is not needed (e.g., internal noise 
is insufficient to make same pairs appear different to such an extent), and so the disparity disappears when RTs for dissimilar "different" pairs are compared with those for asymmetric "same" pairs.

Although this model can account for all the major findings of Experiments 1 and 2, more problematic is the task of explicating the role of lateralized functions beyond merely noting that visual field $x$ response interactions support a dual-process notion. If the right-field advantage for "same" responses is proposed to result from a left-hemisphere superiority in extracting symmetry, for example, then it is difficult to explain why, when "same" responses alone were analyzed, visual field by symmetry interactions were not obtained (since the implication would be that the effect should disappear when pairs were asymmetric). On the other hand, if there are things inherent in "same" and "different" responses per se which are lateralized to the left and right hemispheres, respectively, then it becomes difficult to explain the frequent failure of the same-different factor to interact with visual field (Atkinson \& Egeth, 1973; Egeth, 1971; Moscovitch, Scullion, \& Christie, 1976). Clearly, more research is needed to discover the exact reasons for obtaining such interactions (Bagnara et al., 1982; Cohen, 1973; Egeth \& Epstein, 1972).

\section{REFERENCE NOTE}

1. Boles, D. B. Global versus analytic processing: Is there a hemispheric dichotomy? Manuscript submitted for publication, 1983.

\section{REFERENCES}

Atkinson, J., \& Egeth, H. Right hemisphere superiority in visual orientation matching. Canadian Journal of Psychology, $1973,27,152-158$.

Bagnaha, S., Boles, D. B., Simion, F., \& Umilta, C. Can an analytic/holistic dichotomy explain hemispheric asymmetries? Cortex, 1982, 18, 67-78.

BAmber, D. Reaction times and error rates for same-different judgments of multidimensional stimuli. Perception \& Psychophysics, 1969, 6, 169-174.

Berlucchi, G. Cerebral dominance and interhemispheric communication in normal man. In F. O. Schmitt \& F. G. Worden (Eds.), The neurosciences: Third study program. Cambridge, Mass: M.I.T. Press, 1974.

Bindra, D., Donderi, D. C., \& Nishisato, S. Decision latencies of "same" and "different" judgments. Perception \& Psychophysics, 1968, 3, 121-130.

Boles, D. B., \& Eveland, D. C. Visual and phonetic codes, and the process of generation in letter matching. Journal of Experimental Psychology: Human Perception and Performance, 1983, 9, 657-674.

Cohen, G. Hemispheric differences in serial versus parallel processing. Journal of Experimental Psychology, 1973, 97, 349-356.

Egeth, H. Laterality effects in perceptual matching. Perception \& Psychophysics, 1971, 9, 375-376.

Egeth, H. E., \& Epstein, J. Differential specialization of the cerebral hemispheres for the perception of sameness and difference. Perception \& Psychophysics, 1972, 12, 218-220.

Fairweather, H., Brizzolara, D., Tabossi, P., \& Umilta, C. Functional cerebral lateralization: Dichotomy or plurality? Cortex, 1982, 18, 51-66.
Fox, J. The use of structural diagnostics in recognition. Journal of Experimental Psychology: Human Perception and Performance, 1975, 1, 57-67.

Harvey, L. O. Single representation of the visual midline in humans. Neuropsychologia, 1978, 16, 601-610.

HAUN, F. Functional dissociation of the hemispheres using foveal visual input. Neuropsychologia, 1978, 16, 725-733.

Hellige, J. B., \& WeBster, R. Right hemisphere superiority for initial stages of letter processing. Neuropsychologia, 1979, $17,653-660$.

Hock, H. S. The effects of stimulus structure and familiarity on same-different comparison. Perception \& Psychophysics, 1973, 14, 413-420.

Hodge, D. C. Legibility of a uniform-strokewidth alphabet: I. Relative legibility of upper and lower case letters. Journal of Engineering Psychology, 1962, 1, 34-46.

Klatzky, R. L., \& Atkinson, R. C. Specialization of the cerebral hemispheres in scanning for information in short-term memory. Perception \& Psychophysics, 1971, 10, 335-338.

Knold, J. F., \& Hershe rson, M. Two stages in visual matching. Canadian Journal of Psychology, 1980, 34, 49-61.

Krueger, L. E. A theory of perceptual matching. Psychological Review, 1978, 85, 278-304.

Krueger, L. E. Probing Proctor's priming principle: The effect of simultaneous and sequential presentation on same-different judgments. Journal of Experimental Psychology: Learning, Memory, and Cognition, 1983, 9, 511-523.

Levy-Agresti, J., \& Sperhy, R. W. Differential perceptual capacities in major and minor hemispheres. Proceedings of the National Academy of Sciences U.S.A., 1968, 66, 1151.

Martin, M. Hemispheric specialization for local and global processing. Neuropsychologia, 1979, 17, 33-40.

Moscovitch, M., Scullion, D., \& Christie, D. Early vs. late stages of processing and their relation to functional hemispheric asymmetries in face recognition. Journal of Experimental Psychology: Human Perception \& Performance, 1976, 2, 401-416.

Nickenson, R. S. Binary-classification reaction time: A review of some studies of human information-processing capabilities. Psychonomic Monograph Supplements, 1972, 4(17, Whole No. 65), 275-318.

Nicke rson, R. S. On the time it takes to tell things apart. In J. Requin (Ed.), Attention and performance VII. Hillsdale, N.J: Erlbaum, 1978.

Nickerson, R. S. Context is important but it does not explain everything: A comment on "Matching performance and the similarity structure of the stimulus set" by Crist. Journal of Experimental Psychology: General, 1981, 110, 297-302.

Patterson, K., \& Bradshaw, J. L. Differential hemispheric mediation of nonverbal visual stimuli. Journal of Experimental Psychology: Human Perception \& Performance, 1975, 1, 246-252.

Podgorny, P., \& Garner, W. R. Reaction time as a measure of inter- and intra-object visual similarity: Letters of alphabet. Perception \& Psychophysics, 1979, 26, 37-52.

Posner, M. I. Chronometric explorations of mind. Hillsdale, N.J: Erlbaum, 1978.

Proctor, R. W. A unified theory for matching-task phenomena. Psychological Review, 1981, 88, 291-326.

Richards, J. T. Interitem structure and the facilitation of simultaneous comparison. Journal of Experimental Psychology: Human Perception and Performance, 1978, 4, 72-87.

ROYER, F. L. Detection of symmetry. Journal of Experimental Psychology: Human Perception and Performance, 1981, 7, 1186-1210.

TAYLor, D. A. Holistic and analytic processes in the comparison of letters. Perception \& Psychophysics, 1976, 20, 187-190.

Umilta, C., Salmaso, D., Bagnara, S., \& Simion, F. Evidence for a right hemisphere superiority and for a serial search strategy in a dot detection task. Cortex, 1979, 15, 597-608.

(Manuscript received March 2, 1983; revision accepted for publication September 1, 1983.) 